\title{
Study of Dielectric Constant and Loss Tangent of Ammonium Iron Alum
}

\author{
ARVIND KUMAR RAWAT and TRILOK CHANDRA UPADHYAY
}

Physics Department, H.N.B. Garhwal University, Srinagar (Garhwal), U.K. 246174, India

arvindsgfi@gmail.com

Received 17 September 2015 / Accepted 5 October 2015

\begin{abstract}
With the help of double-time temperature dependent Green's function method and modifying pseudospin-lattice coupled mode model by adding third-and fourth-order phonon anharmonic interactions terms, expressions for ferroelectric mode frequency, dielectric constant and loss tangent have been derived. By fitting model values of physical quantities in above expressions, temperature dependence of above quantities have been calculated numerically. Theoretical results are compared with experimental results of others, which show agreement.
\end{abstract}

Keywords: Green's function, Pseudospin-lattice coupled mode, Anharmonic, Dielectric

\section{Introduction}

Ferroelectric materials have attracted scientists due to their potential applications in production of mall size capacitors of high capacitance, as memory devices for computers, as transducers, and infrared detectors. Alums are interesting materials. Some alums show ferroelectric properties. Ammonium iron alum $\left(\mathrm{NH}_{4}\right) \mathrm{Fe}\left(\mathrm{SO}_{4}\right)_{2} .12 \mathrm{H}_{2} \mathrm{O}$ is ferroelectric below $88 \mathrm{~K}$. Below $88 \mathrm{~K}$, it is cubic while above $88 \mathrm{~K}$ it is paraelectric. In AFeSD alum $\mathrm{NH}_{4}^{+}$group gives rise to order-disorder type of mechanism in proton subsystem associated with these groups. This is assumed to be responsible for the ferroelectric phase transitions in several alums. Due to order-disorder character of the ammonium group in AFeSD, the $H$-bonds associated with these groups undergo some kind of ordering. It then becomes possible to apply pseudospin model similar to the case of KDP system, after suitable modification. The proton motion is associated with the "active" ammonium ion. In this crystal there are very little isotope effect on $\mathrm{T}_{\mathrm{c}}$ and $\mathrm{C}$, the pseudospin motion should be highly damped with strong anharmonic phonon interactions. There has been a considerable interest in the experimental study of AFeSD alum. Derby ${ }^{1}$ has carried out crystal growth of AFeSD alums. Weber ${ }^{2}$ has carried out experimental study of dielectric properties of AFeSD alum. Sachdeva et al. ${ }^{3}$ have done experimental crystal growth studies on AFeSD alum. 
Petrusevki ${ }^{4}$ has carried out vibrational spectra of AFeSD, Selenate and other alums. Gu and $\mathrm{Li}^{5}$ have carried out spectral properties of AFeSD and potassium alum. Gu and $\mathrm{Ho}^{6}$ have done spectral properties studies on AFeSD alum and ammonium alum. Shaxin et al. ${ }^{7}$ have studied application of AFeSD alum in inorganic synthesis.

Bow et al. ${ }^{8}$ have studied experimentally the adjuvant action of AFeSD alums. Earlier theoretical studies on AFeSD alum has been done by O' Reilly and Tsang'. Thereafter Chaudhury et al. ${ }^{10}$ have done theoretical study of AFeSD and MASD alums. Chaudhury et al. ${ }^{10}$ have applied pseudospin-lattice coupled-mode model. These authors have not considered third order phonon anharmonic interaction term. Morever, they have decoupled the correlations at an early stage. As a result some important interactions were disappeared from their calculations. In the present work, we shall modify pseudospin-lattice coupled mode model by adding the third- and fourth-order phonon anharmonic interactions terms. By using double-time thermal Green's function method ${ }^{11}$, expressions for ferroelectric mode frequency, dielectric constant and loss tangent will be obtained. Numerical calculations will be done to obtain thermal dependence of above quantities. Theoretical results will be compared with experimental results of others.

\section{Theory}

For AFeSD alum, Chaudhury et $a l^{10}$ have applied pseudospin-lattice coupled mode model. We shall add third-and fourth-order phonon anharmonic interaction terms:-

$$
\begin{aligned}
& H=-2 \Omega \sum_{i} S_{i}^{x}-\frac{1}{2} \sum_{i j} J_{i j} S_{i}^{Z} S_{j}^{Z}+\frac{1}{4} \sum_{k} \omega_{k}\left(A_{k} A_{k}^{+}+B_{k} B_{k}^{+}\right)-\sum_{i k} V_{i k} S_{i}^{Z} A_{k} \\
& +\sum_{k_{1} k_{2} k_{3}} V^{4}\left(k_{1}, k_{2}, k_{3}\right) A_{k_{1}} A_{k_{2}} A_{k_{3}}+\sum_{k_{1} k_{2} k_{3} k_{4}} V^{4}\left(k_{1}, k_{2}, k_{3}, k_{4}\right) A_{k_{1}} A_{k_{2}} A_{k_{3}} A_{k_{4}} .
\end{aligned}
$$

Following Zubarev ${ }^{13}$, we consider the Green's function

$$
\begin{aligned}
G_{i j}\left(t-t^{\prime}\right) & =\left\langle\left\langle S_{i}^{z}(t) ; S_{j}^{z}\left(t^{\prime}\right)\right\rangle\right\rangle \\
& =-i \theta\left(t-t^{\prime}\right)\left\langle\left[S_{i}^{z}(t) ; S_{j}^{z}\left(t^{\prime}\right)\right]\right\rangle
\end{aligned}
$$

Where $\theta$ is unit step function, $\theta=1$ for $t^{\prime}>t$ and $=0$ for $t^{\prime}<t, \Omega$ is proton tunneling frequency, $S_{i}^{z}$ is spin operator, $\omega_{k}$ is phonon frequency, $A_{k}$ is position operator, $B_{k}$ is momentum operator. Differentiating Eq.(1b) with respect to time $t$ and $t^{\prime}$ two times and setting its Fourier transform into Dyson's equation form

$$
\mathrm{G}(\omega)=\mathrm{G}^{0}(\omega)+\mathrm{G}^{0}(\omega) \tilde{P}(\omega) \mathrm{G}^{0}(\omega)
$$

we obtain Green's function (1b) as

$$
G_{i j}(\omega)=\frac{\Omega\left\langle S_{i}^{x}\right\rangle \delta_{i j}}{\pi\left[\omega^{2}-\hat{\Omega}^{2}-2 i \Omega \Gamma(\omega)\right]}
$$

Where

$$
\hat{\Omega}^{2}=\widetilde{\Omega}^{2}+\Delta(\omega),
$$




$$
\begin{aligned}
\widetilde{\Omega}^{2} & =a^{2}+b^{2}-b c, \\
a & =J\left\langle S_{i}^{z}\right\rangle, \\
b & =2 \Omega \\
c & =J\left\langle S^{x}\right\rangle
\end{aligned}
$$

and $\Delta(\omega)$ and $\Gamma(\omega)$ are obtained as

$$
\begin{aligned}
& \Delta(\omega)=\frac{a^{4}}{2 \Omega\left(\omega^{2}-\tilde{\Omega}^{2}\right)}+\frac{b^{2} c^{2}}{2 \Omega\left(\omega^{2}-\tilde{\Omega}^{2}\right)}+\frac{V_{i k}^{2} N_{K} a^{2}}{2 \Omega\left(\omega^{2}-\tilde{\Omega}^{2}\right)}+\frac{2 V_{i k}^{2}\left\langle S_{1 i}^{x}\right\rangle \omega_{k} \delta_{k-k^{\prime}}\left(\omega^{2}-\tilde{\tilde{\omega}}_{k}^{2}\right)}{\left(\omega^{2}-\tilde{\tilde{\omega}}_{k}^{2}\right)+4 \omega_{k}^{2} \Gamma_{k}^{2}(\omega)}, \\
& \Gamma(\omega)=\frac{\pi a^{4}}{4 \Omega \tilde{\Omega}}[\delta(\omega-\tilde{\Omega})-\delta(\omega+\tilde{\Omega})]+\frac{\pi V_{i k}^{2} N_{k} a^{2}}{4 \Omega \tilde{\Omega}}[\delta(\omega-\tilde{\Omega})-\delta(\omega+\tilde{\Omega})]+\frac{2 V_{i k}^{2}\left\langle S_{1 i}^{x}\right\rangle \omega_{k} \delta_{k-k^{\prime}}\left(\omega^{2}-\tilde{\tilde{\omega}}_{k}^{2}\right)}{\left(\omega^{2}-\tilde{\tilde{\omega}}_{k}^{2}\right)+4 \omega_{k}^{2} \Gamma_{k}^{2}(\omega)}
\end{aligned}
$$

We obtain susceptibility $\chi$ from Green's function (2) similarly as since

$$
\chi=-\lim _{x \rightarrow 0} 2 \pi \chi N \mu^{2} G_{i j}(\omega)
$$

$\mathrm{N}$ is no of dipoles per unit volume and $\mu$ is dipole moment associated with $\mathrm{O}-\mathrm{H}$...... bond. We know that dielectric constant $\boldsymbol{\epsilon}$ is related to susceptibility $\chi$ as

$$
\epsilon=1+4 \pi \chi
$$

or simply $\in=4 \pi \chi$ as $\in>>1$. From Eq. (8), (9) and (2) we obtain

$$
\epsilon=-\frac{8 \pi N \mu^{2} \Omega\left\langle S_{i}^{X}\right\rangle\left\{\left(\omega^{2}-\hat{\Omega}^{2}\right)+2 \Omega i \Gamma(\omega)\right\}}{\left[\left(\omega^{2}-\hat{\Omega}^{2}-2 \Omega^{2} \Gamma^{2}(\omega)\right)\right]} .
$$

The tangent loss is obtained from Eq.(10)

$$
\tan (\delta)=\frac{\epsilon^{\prime \prime}}{\epsilon}=-\frac{2 \Omega \Gamma(\omega)}{\left(\omega^{2}-\hat{\Omega}^{2}\right)} \mathrm{n}
$$

From Eqs. (10) and (11) one observes that dielectric constant and loss tangent depend on modified soft mode frequency. Hence these depend on tunneling frequency as well as on anharmonic interactions terms.

\section{Results and Discussion}

For AFeSD alum, we use the model values of physical quantities ${ }^{11}$ as $\Omega=0.25, T_{C}=88 \mathrm{~K}$, $J^{\prime}=244.64 \mathrm{~cm}^{-1}, V_{i k}=6.49 \mathrm{~cm}^{-1}, N_{k}=0.512 \times 10^{21}, \mu \times 10^{18} \mathrm{cgs}=1.405, \omega_{k}=5 \mathrm{~cm}^{-1}, C=425 \mathrm{~K}$, $J=183.49 \mathrm{~cm}^{-1}, A_{k} \times 10^{-17}\left(\operatorname{ergK}^{-1}\right)=0.05$. We calculate the temperature dependence of ferroelectric mode frequency $\hat{\Omega}, \in$ and $\tan \delta$ using Eq. (3), (10) and (11) and above model values. The results have been shown in Figures 1, 2 and 3 respectively. Theoretical results have been compared with experimental results of Pepinsky et al. ${ }^{12}$ for dielectric constant and correlated results for ferroelectric frequency and tangent loss for AFeSD crystal. Our theoretical results agree with experimental results of Pepinsky et al. ${ }^{12}$. From Eq. (3) and Figure 1, it is observed that ferroelectric mode frequency decreases as we approach from low temperature side towards Curie temperature. At Curie temperature frequency becomes infinitesimally small, and increases above it. Our finding is in agreement with experimental 
observations. Our Eq. (10) and Figure 2 show that dielectric constant first increases as we increase temperature from low temperature side becoming anomalously large at transition temperature. Above $T_{c}$ dielectric constant decreases with increasing temperature. Our findings agree with experimental results of Pepinsky et al. ${ }^{12}$ observations. Similar behaviour is predicted by our Eq. (11) and Figure 3 for AFeSd alum.

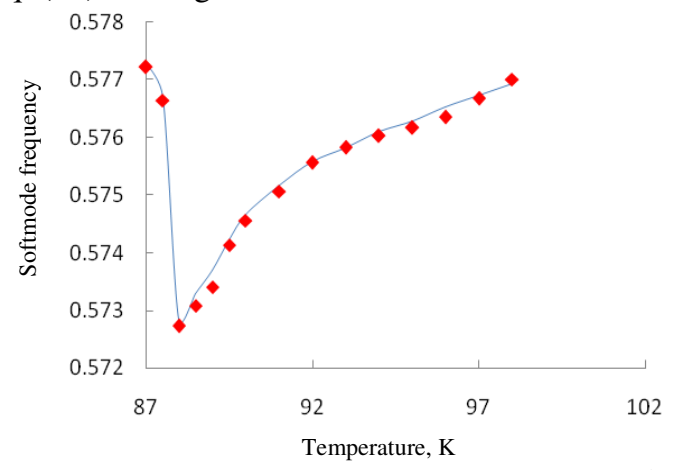

Figure 1. Temperature dependence of soft mode frequency $\Omega\left(\mathrm{cm}^{-1}\right)$ of AFeSD alum (-our

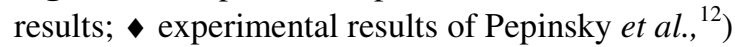

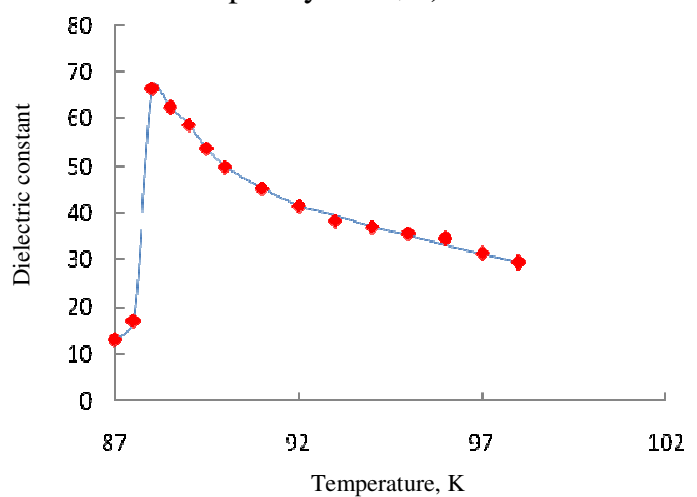

Figure 2. Temperature dependence of dielectric constant $\epsilon$ of AFeSD alum ( - our results; - experimental results of Pepinsky et al., ${ }^{12}$ )

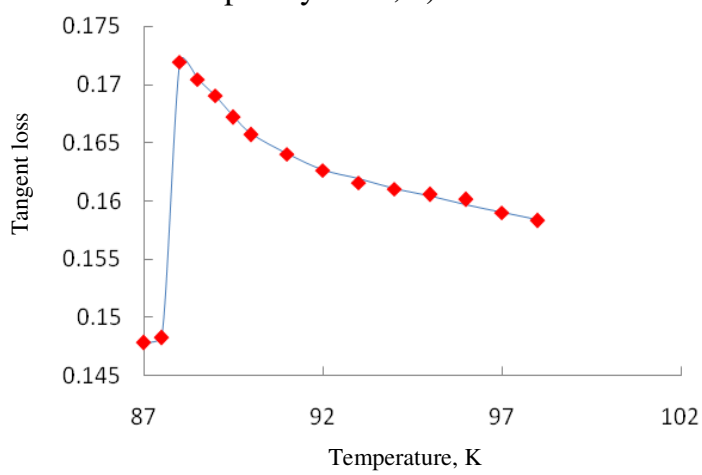

Figure 3. Temperature dependence of tangent loss $(\delta)$ of AFeSD alum (- our results; - experiment results of Pepensky et al. ${ }^{12}$.) 


\section{Conclusion}

As can be understood from the above results, the pseudospin-lattice coupled mode model along with thirsd-and fourth- order phonon anharmonic interactions terms explains the dielectric properties and ferroelectric behaviour of AFeSD alum clearly. The phonon anharmonic interaction terms significantly affect the temperature dependence of softmode frequency, dielectric constant and loss tangent in AFeSD alum. Our results are better than the results of earlier authors", quantitatively.

\section{Acknowledgement}

The authors are thankful to Eminent Prof B S Semwal (Former, HOD Physics) for his kind blessings and to his interest in the work. Dr G. M. Kalamse, Science College, Nanded (M.S.) for encouragement, they are thankful to Prof Lalitha Siredeshmukh (K.U., Warangal), Prof K G Subhadra (K U, Warangal), Prof Kamal Singh (Nagpur Univ \& Amrawati Univ) Prof N S Negi (H P Univ Shimla) and Prof S C Bhatt (HOD, \& Convener Physics, HNBGU Srinagar (Garhwal)) and Dr M Uniyal (HNBGU Srinagar (garhwal)) for their encouragements.

\section{References}

1. Derby J J, $14^{\text {th }}$ Int' Summer School on Crystal Growth, Dalian, China, Aug, 2010, 1-7.

2. Weber, Zeits fur Kristall, 2010, 1149, 269.

3. Sachdeva A, Singh R, Sing P K and Bhattacharya B, Mater Technol., 2013, 47(4), 467-471.

4. Petrusevki V M, Macedonian J Chem Chem Engg., 2015, 34(1), 73-85.

5. $\mathrm{Gu}, \mathrm{H}$ and $\mathrm{Li} \mathrm{Y}$, Spectrochimia Acta Part A, 2015, 139, 342-345; DOI:10.1016/j.saa.2014.12.087

6. Gu H and Hao X, 2015, 146, 273-276; Spectrochimia Acta Part A, DOI:10.1016/j.saa.2015.03.066

7. Shaxin Yu, et al., General Review, 2005, 17(1), 27-30.

8. Mbow L M, Ennio Gregorio De and Ulmer Jeffrey B, Nature Medicine, 2011, 17(4), 415-416; DOI:10.1038/nm0411-415

9. Reilly O D E and Tsang T, Phys Rev., 1967, 157, 417.

10. Chaudhury K R, Nath D, Banerjee S and Chaudhuri B K, Phys Rev B, 1982, 26, 6276.

11. Zubarev D N, Sov Phys Usp., 1960, 3(3), 320.

12. Pepinsky R, Jona S and Shirane G, Phys Rev., 1956, 102, 1181-1182. 